\title{
Infinitely many universally tight contact manifolds with trivial Ozsváth-Szabó contact invariants
}

\author{
PAOLO GHIGGINI
}

\begin{abstract}
In this article we present infinitely many 3-manifolds admitting infinitely many universally tight contact structures each with trivial Ozsváth-Szabó contact invariants. By known properties of these invariants the contact structures constructed here are non weakly symplectically fillable.
\end{abstract}

57R17; 57R57

\section{Introduction}

Recently Ozsváth and Szabó introduced a new isotopy invariant $c(\xi)$ for contact 3manifolds $(Y, \xi)$ belonging to the Heegaard Floer homology group $\widehat{H F}(-Y)$. They proved [27] that $c(\xi)=0$ if $\xi$ is an overtwisted contact structure, and that $c(\xi) \neq 0$ if $\xi$ is Stein fillable. Later, they introduced also a refined version of the contact invariant denoted by $\underline{c}(\xi)$ taking values in the so-called Heegaard Floer homology group with twisted coefficients. They proved [24, Theorem 4.2] that $\underline{c}(\xi) \neq 0$ if $(Y, \xi)$ is weakly fillable.

The Ozsváth-Szabó contact invariants have been successfully used to prove tightness for several manifolds which had resisted to any previously known technique: see the papers $[19 ; 17 ; 18]$ by Lisca and Stipsicz. This fact raised the hope that these invariants could be non trivial for any tight contact structure. However in [6] we showed that the untwisted contact invariant reduced modulo 2 can vanish even for weakly symplectically fillable contact structures. Those examples however left open the question whether the twisted invariants were non trivial for every tight contact structures. In this article we give the following negative answer.

Theorem 1.1 For any choice of coefficients $r_{i} \in(0,1) \cap \mathbb{Q}$ the Seifert manifold $M\left(r_{1}, r_{2}, r_{3}, r_{4}\right)$ defined by the surgery diagram in Figure 1 admits infinitely many pairwise non isomorphic universally tight contact structures with trivial Ozsváth-Szabó contact invariants. 


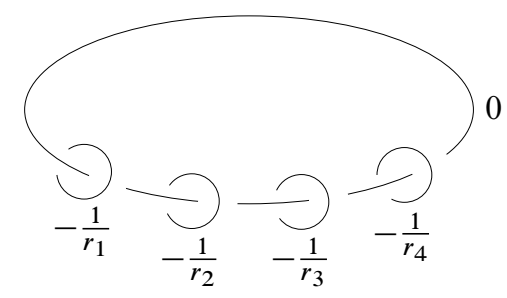

Figure 1: The surgery diagram of $M\left(r_{1}, r_{2}, r_{3}, r_{4}\right)$

Although Theorem 1.1 will be proved for the untwisted invariant, it holds for the twisted ones as well because $M\left(r_{1}, r_{2}, r_{3}, r_{4}\right)$ is a rational homology sphere for our choice of Seifert coefficients, and for rational homology spheres the twisted and the untwisted contact invariants coincide. The following corollary is therefore a consequence of the non triviality of the twisted invariant for weakly symplectically fillable contact structures [24, Theorem 4.2], and of the fact that $H_{1}\left(M\left(r_{1}, r_{2}, r_{3}, r_{4}\right), \mathbb{Q}\right)=0$.

Corollary 1.2 For any choice of coefficients $r_{i} \in(0,1) \cap \mathbb{Q}$ the Seifert manifold $M\left(r_{1}, r_{2}, r_{3}, r_{4}\right)$ admits infinitely many pairwise non isomorphic universally tight contact structures which are not weakly symplectically fillable.

This corollary provides the first universally tight contact structures which are known to be non weakly symplectically fillable, therefore it answers negatively to a question [5, Question 4] of Etnyre and Ng, asking whether any universally tight contact 3-manifold has to be weakly fillable.

Remark 1.3 The non fillable contact structures constructed here are all homotopic to Stein fillable contact structures. Our construction contrasts with all previous non fillability results, whose proofs relied on homotopic properties of the contact structures.

\section{Acknowledgements}

This work was initiated while the author was visiting Princeton University supported by the NSF Focused Research Grant FRG-024466. We would like to thank András Stipsicz for several useful discussions about Heegaard Floer homology, and Steve Boyer for his help with the diffeomorphisms of Seifert manifolds. The author was partially supported by a CIRGET fellowship and by the Chaire de Recherche du Canada en algèbre, combinatoire et informatique mathématique de l'UQAM. 


\section{Construction of the contact structures}

In this section for any natural number $n$ we will construct a universally tight contact structure $\xi_{n}$ on $M\left(r_{1}, r_{2}, r_{3}, r_{4}\right)$.

We denote by $M^{\prime}\left(r_{1}, \ldots, r_{k}\right)$ the Seifert manifold over $D^{2}$ with $k$ singular fibres with Seifert coefficients $r_{1}, \ldots, r_{k}$. We can decompose the manifold $M\left(r_{1}, r_{2}, r_{3}, r_{4}\right)$ as

$$
M\left(r_{1}, r_{2}, r_{3}, r_{4}\right)=M^{\prime}\left(r_{1}, r_{2}\right) \cup T^{2} \times[-1,1] \cup M^{\prime}\left(r_{3}, r_{4}\right) .
$$

The orientation on $T^{2} \times\{-1\}$ is given by the inward normal convention, while the orientation on $T^{2} \times\{1\}$ is given by the outward normal convention, therefore $\partial M^{\prime}\left(r_{1}, r_{2}\right)$ is identified to $T^{2} \times\{-1\}$, and $T^{2} \times\{1\}$ is identified to $-\partial M^{\prime}\left(r_{3}, r_{4}\right)$.

Since our construction will not use the Seifert coefficients of the fibres in any specific way, except for the fact $r_{i} \in(0,1)$, we will suppress them from the notation, and will call $M=M\left(r_{1}, r_{2}, r_{3}, r_{4}\right), M_{1}^{\prime}=M^{\prime}\left(r_{1}, r_{2}\right)$, and $M_{2}^{\prime}=M^{\prime}\left(r_{3}, r_{4}\right)$.

By Hatcher [11, Proposition 2.2 and Section 1.2], $M_{i}^{\prime}$ is a surface bundle over $S^{1}$. Let $\Sigma_{i}$ be the fibre, and $\phi_{i}$ be the monodromy of this bundle. The Seifert fibration on $M_{i}^{\prime}$ restricted to $\Sigma_{i}$ is a branched cover $\Sigma_{i} \rightarrow D^{2}$ with finite fibre, and $\phi_{i}$ is a deck transformation, therefore $\phi_{i}$ has finite order $n_{i}$.

Call $\mathcal{C}_{i}$ the set of all 1 -forms $\beta$ over $\Sigma_{i}$ such that

(1) $d \beta$ is a volume form on $\Sigma_{i}$,

(2) $\left.\beta\right|_{\partial \Sigma_{i}}$ is a volume form on $\partial \Sigma_{i}$.

By Thurston-Winkelnkemper [29] the sets $\mathcal{C}_{i}$ are nonempty and convex. We define a $\phi_{i}$-invariant $1-$ form $\bar{\beta}_{i}$ on $\Sigma_{i}$ by averaging as follows: we pick any 1 -form $\beta_{i} \in \mathcal{C}_{i}$ and define

$$
\bar{\beta}_{i}=\frac{1}{n_{i}} \sum_{k=0}^{n_{i}-1}\left(\phi_{i}^{k}\right)^{*} \beta .
$$

By the convexity of $\mathcal{C}_{i}$ we have $\bar{\beta}_{i} \in \mathcal{C}_{i}$. If $t$ is the coordinate of $[0,1]$, for any $K>0$ the 1 -form $d t+K \bar{\beta}_{i}$ is a contact form on $\Sigma_{i} \times[0,1]$ which gives a well defined contact form on $M_{i}^{\prime}$. By Gray's Theorem the contact structures on $M_{i}^{\prime}$ obtained from different choices of $\beta_{i}$ are isotopic, while the actual value of $K$ has no relevance for our construction. We denote by $\xi_{+}$the kernel of $d t+K \bar{\beta}_{i}$, and by $\xi_{-}$the kernel of $-\left(d t+K \bar{\beta}_{i}\right)$. The following lemma is a straightforward computation on the contact forms. 
Lemma 2.1 $\partial M_{i}^{\prime}$ is a prelagrangian torus with slope $s_{i}$ with respect to $\xi_{+}$and $\xi_{-}$. We can chose $K$ so that $-2<s_{1}<-\left(r_{1}+r_{2}\right)$ and $-2<s_{2}<-\left(r_{3}+r_{4}\right)$. The Reeb vector fields of the contact forms $\pm\left(d t+K \bar{\beta}_{i}\right)$ are tangent to the fibres of the Seifert fibration of $M_{i}^{\prime}$, and $\xi_{+}$and $\xi_{-}$are transverse to the Seifert fibration.

In the following we will always assume that $K$ has been chosen so that the inequalities in Lemma 2.1 hold.

On $T^{2} \times[-1,1]$ we consider the contact structures

$$
\alpha_{n}\left(s_{1}, s_{2}\right)=\operatorname{ker}\left(\cos \left(\varphi_{n}(t)\right) d x+\sin \left(\varphi_{n}(t)\right) d y\right)
$$

for a smooth function $\varphi_{n}:[-1,1] \rightarrow \mathbb{R}$ such that

(1) $\varphi_{n}^{\prime}(t)>0$ for any $t \in[-1,1]$,

(2) $\left[\frac{\varphi_{n}(1)-\varphi_{n}(-1)}{\pi}\right]=n$,

(3) $T^{2} \times\{-1\}$ has slope $s_{1}$ and $T^{2} \times\{1\}$ has slope $-s_{2}$,

(4) $\varphi_{n}(-1) \in\left(0, \frac{\pi}{2}\right)$.

Condition (1) implies that $\alpha_{n}\left(s_{1}, s_{2}\right)$ is a contact structure, condition (2) implies that it has twisting $n \pi$ in the sense of Honda [13, Section 2.2.1], and condition (4) is simply a normalisation condition. The set of functions satisfying these conditions is convex, therefore by Gray's Theorem the isotopy type of $\alpha_{n}\left(s_{1}, s_{2}\right)$ depends only on $n, s_{1}$ and $s_{2}$.

Definition 2.2 We define the contact structures $\xi_{n}$ on $M\left(r_{1}, r_{2}, r_{3}, r_{4}\right)$ so that they coincide with $\xi_{+}$on $M_{1}^{\prime}$, with $\xi_{-}$on $M_{2}^{\prime}$, and with $\alpha_{2 n}\left(s_{1}, s_{2}\right)$ on $T^{2} \times[-1,1]$, where $s_{i}$ is the boundary slope of $M_{i}^{\prime}$.

Following Colin and Honda [2] we say that a contact structure is hypertight if it can be defined by a contact form whose associated Reeb vector field has no contractible periodic orbits. By Hofer [12, Theorem 1] hypertight contact structures are tight.

Theorem 2.3 The contact structures $\xi_{n}$ on $M\left(r_{1}, r_{2}, r_{3}, r_{4}\right)$ are hypertight for any $n \geq 0$.

Proof By [2, Lemma 9.1] we can isotope $\xi_{+}$and $\xi_{-}$relative to the boundary, so that they are defined by contact 1 -forms which glue to the contact form of $\alpha_{2 n}\left(s_{1}, s_{2}\right)$ to give a globally defined contact form for $\xi_{n}$. Moreover, the isotopy can be chosen so that the Reeb vector fields of $\xi_{+}$and $\xi_{-}$remain transverse to the fibrations over $S^{1}$ defined on $M_{1}^{\prime}$ and $M_{2}^{\prime}$. 
Any periodic orbit of the Reeb flow must be completely contained in one of the three pieces $M_{1}^{\prime}, M_{2}^{\prime}$ and $T^{2} \times[-1,1]$ in which $M$ has been decomposed, because the Reeb vector field is tangent to $\partial M_{1}^{\prime}$ and to $\partial M_{2}^{\prime}$. Moreover, the inclusions $M_{i}^{\prime} \hookrightarrow M$ and $T^{2} \times[-1,1] \hookrightarrow M$ induce injective maps between the fundamental groups, therefore a periodic orbit of the Reeb flow is contractible in $M$ if and only it is contractible in the piece it is contained in. This implies that there are no contractible periodic orbits of the Reeb flow in $M$, because the Reeb vector field is transverse to the $S^{1}$-fibrations in $M_{i}^{\prime}$, and in $T^{2} \times[-1,1]$ its closed orbits are homotopically non trivial in the incompressible tori $T^{2} \times\{c\}$.

In order to prove universal tightness for $\xi_{n}$ we need the following lemma about the coverings of Seifert manifolds.

Lemma 2.4 Let $M$ be a Seifert manifold with base $B$, and denote its universal covering by $\widetilde{M}$. If $K$ is a compact set of $\widetilde{M}$, then there exists a finite covering $\bar{M}$ of $M$ for which the projection $\widetilde{M} \rightarrow \bar{M}$ is injective on $K$.

Proof We have to consider only the case when the universal cover is not already a finite cover itself. Consider the orbifold structure induced on $B$ by the Seifert fibration on $M$. Either $M$ has at least three singular fibres, or the genus of $B$ (as a surface) is $g>0$ because we have assumed that the universal cover of $M$ is infinite. In these cases $B$ is a good orbifold, therefore there is a finite orbifold covering $B^{\prime} \rightarrow B$ such that $B^{\prime}$ has no singular points: see Scott [28, Theorem 2.3 and Theorem 2.5].

We pull back the Seifert fibration of $M$ to $B^{\prime}$ in order to obtain a Seifert manifold $M^{\prime}$ which fibres over $B^{\prime}$ and a finite covering $M^{\prime} \rightarrow M$. The Seifert fibration on $M^{\prime}$ has no singular fibres because $B^{\prime}$ has no singular points, therefore $M^{\prime}$ is a circle bundle over $B^{\prime}$. This concludes the proof because the lemma holds trivially for circle bundles over surfaces, and the composition of finite coverings is still a finite covering.

Corollary 2.5 The contact structures $\xi_{n}$ on $M\left(r_{1}, r_{2}, r_{3}, r_{4}\right)$ are universally tight for all $n \geq 0$.

Proof Suppose by contradiction that the universal cover of $\left(M, \xi_{n}\right)$ contains an overtwisted disc. Since the overtwisted disc is compact, by Lemma $2.4\left(M, \xi_{n}\right)$ has an overtwisted finite cover. This is a contradiction because any finite cover of a hypertight contact manifold is hypertight again, and therefore it is tight. 


\section{Decomposition of the tight contact structures}

Let $M$ be a Seifert manifold with base $B$ and $k$ singular fibres $F_{1}, \ldots, F_{k}$, and let $U_{i}$ be a standard neighbourhood of $F_{i}, i=1, \ldots, k$. Then $M \backslash \bigcup_{i=1}^{k} U_{i}$ can be identified with $S \times S^{1}$ where $S$ is the surface obtained by removing $k$ disjoint discs centred at the images of the singular fibres from the base $B$. This diffeomorphism determines identifications of $-\partial\left(M \backslash U_{i}\right)$ with $\mathbb{R}^{2} / \mathbb{Z}^{2}$ so that $\left(\begin{array}{l}1 \\ 0\end{array}\right)$ is the direction of the section $S \times\{1\}$ and $\left(\begin{array}{l}0 \\ 1\end{array}\right)$ is the direction of the regular fibres. In order to fix one among the infinitely many product structures on $M \backslash \bigcup_{i=1}^{k} U_{i}$ we also require the meridian of each $U_{i}$ to have slope $-\frac{\beta_{i}}{\alpha_{i}}$ in $-\partial\left(M \backslash V_{i}\right)$ with $\frac{\beta_{i}}{\alpha_{1}}=r_{1}$.

We also choose an identification between $\partial U_{i}$ and $\mathbb{R}^{2} / \mathbb{Z}^{2}$ so that $\left(\begin{array}{l}1 \\ 0\end{array}\right)$ is the direction of the meridian of $U_{i}$ and $\left(\begin{array}{l}0 \\ 1\end{array}\right)$ is the direction of a longitude. Notice that $\partial U_{i}$ and $-\partial\left(M \backslash U_{i}\right)$ coincide as sets, but are identified with $\mathbb{R}^{2} / \mathbb{Z}^{2}$ in different ways. We can choose the longitude on $U_{i}$ so that these two identifications are related by gluing matrices $A_{i}: \partial U_{i} \rightarrow-\partial\left(M \backslash U_{i}\right)$ given by

$$
A_{i}=\left(\begin{array}{cc}
\alpha_{i} & \alpha_{i}^{\prime} \\
-\beta_{i} & -\beta_{i}^{\prime}
\end{array}\right)
$$

with $\beta_{i} \alpha_{i}^{\prime}-\alpha_{i} \beta_{i}^{\prime}=1$ and $0<\alpha_{i}^{\prime}<\alpha_{i}$.

Lemma 3.1 Let $\xi$ be a tight contact structure on $M$, and assume that $(M, \xi)$ has a Legendrian regular fibre $L$ with twisting number 0 (possibly after isotopy), which means that its contact framing coincides with the framing determined by the fibration. Then for $i=1, \ldots, k$ there exist tubular neighbourhoods $V_{i}$ of the singular fibres $F_{i}$ so that $\partial\left(M \backslash V_{i}\right)$ is a convex torus in standard form with infinite slope.

Proof Make the singular fibres $F_{i}$ Legendrian with very low twisting numbers $n_{i}<0$, and consider their standard neighbourhoods $U_{i}$ for $i=1, \ldots, k$. Without loss of generality we can assume $L \cap U_{i}=\varnothing$ for $i=1, \ldots, k$. Let $A_{i}$ be a convex vertical annulus between $L$ and a Legendrian ruling curve of $\partial\left(M \backslash U_{i}\right)$. By the Imbalance Principle, Honda [13, Proposition 3.17], $A_{i}$ produces a bypass attached to $-\partial\left(M \backslash U_{i}\right)$ along a vertical Legendrian ruling curve. Then using the bypass attachment Lemma [13, Lemma 3.15] we can thicken $U_{i}$ until we obtain a convex solid torus $V_{i}$ such that $-\partial\left(M \backslash V_{i}\right)$ has infinite slope.

We call ( $\left.M \backslash \bigcup V_{i},\left.\xi\right|_{M \backslash \cup V_{i}}\right)$ the background of $(M, \xi)$. Since the background of $(M, \xi)$ has infinite boundary slopes, by Honda [14, Section 4.3] it is isomorphic to 
an $S^{1}$-invariant tight contact structure $\left(S \times S^{1}, \xi_{\Gamma_{S_{0}}}\right)$. Here $\xi_{\Gamma_{S_{0}}}$ denotes the $S^{1}-$ invariant contact structure on $S \times S^{1}$ inducing the dividing set $\Gamma_{S_{0}}$ on a convex \#Гminimising section $S_{0} \subset S \times S^{1}$ with Legendrian boundary. By [14, Proposition 4.4] the isotopy class of $\xi_{\Gamma_{S_{0}}}$ is completely determined by $\Gamma_{S_{0}}$.

Let $c_{1}$ be the smallest number for which the torus $T^{2} \times\left\{c_{1}\right\}$ in the contact manifold $\left(T^{2} \times[-1,1], \alpha_{2 n}\left(s_{1}, s_{2}\right)\right)$ has infinite slope, and let $c_{2}$ be the biggest one. Also, let $c_{1}^{\prime}$ be the first number for which $T^{2} \times\left\{c_{1}^{\prime}\right\}$ has slope -2 and let $c_{2}^{\prime}$ be the last number for which $T^{2} \times\left\{c_{2}^{\prime}\right\}$ has slope 2. $c_{1}^{\prime}$ and $c_{2}^{\prime}$ exist because of Lemma 2.1. Call

$$
\begin{aligned}
& \left(M_{c_{1}^{\prime}}, \widetilde{\xi}_{+}^{\prime}\right)=\left(M_{1}^{\prime}, \xi_{+}\right) \cup\left(T^{2} \times\left[-1, c_{1}^{\prime}\right],\left.\alpha_{2 n}\left(s_{1}, s_{2}\right)\right|_{T^{2} \times\left[-1, c_{1}^{\prime}\right]}\right) \\
& \left(M_{c_{2}^{\prime}}, \tilde{\xi}_{-}^{\prime}\right)=\left(M_{2}^{\prime}, \xi_{-}\right) \cup\left(T^{2} \times\left[c_{2}^{\prime}, 1\right],\left.\alpha_{2 n}\left(s_{1}, s_{2}\right)\right|_{T^{2} \times\left[c_{2}^{\prime}, 1\right]}\right) \\
& \left(M_{c_{1}}, \tilde{\xi}_{+}\right)=\left(M_{1}^{\prime}, \xi_{+}\right) \cup\left(T^{2} \times\left[-1, c_{1}\right],\left.\alpha_{2 n}\left(s_{1}, s_{2}\right)\right|_{T^{2 \times\left[-1, c_{1}\right]}}\right) \\
& \left(M_{c_{2}}, \tilde{\xi}_{-}\right)=\left(M_{2}^{\prime}, \xi_{-}\right) \cup\left(T^{2} \times\left[c_{2}, 1\right],\left.\alpha_{2 n}\left(s_{1}, s_{2}\right)\right|_{T^{2} \times\left[c_{2}, 1\right]}\right) .
\end{aligned}
$$

Lemma 3.2 $\left(M_{c_{1}^{\prime}}, \tilde{\xi}_{+}^{\prime}\right)$ and $\left(M_{c_{2}^{\prime}}, \tilde{\xi}_{-}^{\prime}\right)$ contain no Legendrian curves with twisting number 0 isotopic to regular fibres.

Proof We prove the lemma only for $\left(M_{c_{1}^{\prime}}, \widetilde{\xi}_{+}^{\prime}\right)$ because the proof for $\left(M_{c_{2}^{\prime}}, \widetilde{\xi}_{-}^{\prime}\right)$ is the same.

Fix a rational number $r_{3}^{\prime}<-2$, and consider the matrix with integral entries

$$
A\left(r_{3}^{\prime}\right)=\left(\begin{array}{cc}
\alpha & \alpha^{\prime} \\
-\beta & -\beta^{\prime}
\end{array}\right)
$$

such that $r_{3}^{\prime}=\frac{\beta}{\alpha}, \alpha^{\prime} \beta-\alpha \beta^{\prime}=1$, and $0<\alpha^{\prime}<\alpha$. Applying $A\left(r_{3}^{\prime}\right)^{-1}$ to $-\partial M_{c_{1}^{\prime}}$ we obtain a prelagrangian torus with slope

$$
-\frac{\beta+2 \alpha}{\beta^{\prime}+2 \alpha^{\prime}}=-\frac{\alpha}{\alpha^{\prime}-\frac{1}{\beta+2 \alpha}}
$$

which is greater than the slope of the Seifert fibration $-\frac{\alpha}{\alpha^{\prime}}$ because $\frac{\beta}{\alpha}<-2$.

Put polar coordinates $(\rho, \theta)$ on $\mathbb{R}^{2}$, and call

$$
D\left(\rho_{0}\right)=\left\{(\rho, \theta) \in \mathbb{R}^{2}: \rho \leq \rho_{0}\right\} .
$$

We can choose $\rho_{0}$ so that $\left(D\left(\rho_{0}\right) \times S^{1}, \operatorname{ker}\left(d z-\rho^{2} d \theta\right)\right)$ has prelagrangian boundary and boundary slope

$$
-\frac{\beta+2 \alpha}{\beta^{\prime}+2 \alpha^{\prime}}
$$


If we glue the tight solid torus

$$
\left(D\left(\rho_{0}\right) \times S^{1}, \operatorname{ker}\left(d z-\rho^{2} d \theta\right)\right)
$$

to $-\partial M_{c_{1}^{\prime}}$ by the map $A\left(r_{3}^{\prime}\right)$, we obtain a contact structure $\tau$ on $M\left(r_{1}, r_{2}, r_{3}^{\prime}\right)$. This contact structure is transverse to the Seifert fibration because the contact planes do not twist enough to become tangent to the fibres.

Transverse contact structures in Seifert manifolds are tight by Lisca-Matić [16, Corollary 2.2], therefore $\mathrm{Wu}$ [30, Theorem 1.4] implies that $\left(M\left(r_{1}, r_{2}, r_{3}^{\prime}\right), \tau\right)$ contains no Legendrian curve with twisting number 0 isotopic to a regular fibre. Consequently, $\left(M_{c_{1}^{\prime}}, \tilde{\xi}_{+}^{\prime}\right)$ contains no such a curve either.

Lemma 3.3 For any $i=1,2,3,4$ there exists a tubular neighbourhood $V_{i}^{\prime}$ of the singular fibre $F_{i}$ such that $V_{1}^{\prime}, V_{2}^{\prime} \subset M_{c_{1}^{\prime}}, V_{3}^{\prime}, V_{4}^{\prime} \subset M_{c_{2}^{\prime}}$, and $-\partial\left(M \backslash V_{i}^{\prime}\right)$ has slope -1 . Moreover there exist collars $C_{1}$ of $\partial M_{c_{1}}$ in $M_{c_{1}} \backslash\left(V_{1}^{\prime} \cup V_{2}^{\prime}\right)$ and $C_{2}$ of $\partial M_{c_{2}}$ in $M_{c_{2}} \backslash\left(V_{3}^{\prime} \cup V_{4}^{\prime}\right)$ such that $\partial\left(M_{c_{1}} \backslash C_{1}\right)$ and $\partial\left(M_{c_{2}} \backslash C_{2}\right)$ are convex tori with slope -1 .

Proof Again, we prove the lemma only for $M_{c_{1}^{\prime}}$. We perturb $\partial M_{c_{1}^{\prime}}$ so that it becomes a convex torus in standard form with vertical ruling, then we make the singular fibres $F_{1}$ and $F_{2}$ Legendrian with very low twisting numbers $k_{1}$ and $k_{2}$, and take standard neighbourhoods $U_{i}$ of $F_{i}$. The slopes of $-\partial\left(M_{c_{1}^{\prime}} \backslash\left(U_{1} \cup U_{2}\right)\right)$ are

$$
\left\{\begin{array}{cl}
-\frac{k_{1} \beta_{1}+\beta_{1}^{\prime}}{k_{1} \alpha_{1}+\alpha_{1}^{\prime}} & \text { on the component corresponding to } \partial U_{1}, \\
-\frac{k_{2} \beta_{2}+\beta_{2}^{\prime}}{k_{2} \alpha_{2}+\alpha_{2}^{\prime}} & \text { on the component corresponding to } \partial U_{2}, \\
2 & \text { on }-\partial M_{c_{1}^{\prime}} .
\end{array}\right.
$$

We can make $-\frac{k_{i} \beta_{i}+\beta_{i}^{\prime}}{k_{i} \alpha_{i}+\alpha_{i}^{\prime}}$ arbitrarily close to $-r_{i} \in(-1,0)$ by making $k_{i}$ very large in absolute value.

Take convex vertical annuli $A_{i}$ between a vertical Legendrian ruling curve of $\partial M_{c_{1}^{\prime}}$ and a vertical Legendrian ruling curve of the component of $\partial\left(M_{c_{1}^{\prime}} \backslash\left(U_{1} \cup U_{2}\right)\right)$ corresponding to $\partial U_{i}$ for $i=1,2$. By the imbalance principle of Honda [13, Proposition 3.17] $A_{i}$ carries a bypass on the side of $\partial U_{i}$. Attaching this bypass we can thicken $U_{i}$ and reduce the slope of the component of $-\partial\left(M_{c_{1}^{\prime}} \backslash\left(U_{1} \cup U_{2}\right)\right)$ corresponding to $\partial U_{i}$. We can repeat this procedure until we get neighbourhoods $V_{1}^{\prime}$ and $V_{2}^{\prime}$ of $F_{1}$ and $F_{2}$ such that the slopes of $-\partial\left(M_{c_{1}^{\prime}} \backslash\left(V_{1}^{\prime} \cup V_{2}^{\prime}\right)\right)$ are $-1,-1$, and 2 . Denote by $T_{1}$ and $T_{2}$ the components of $-\partial\left(M_{c_{1}^{\prime}} \backslash\left(V_{1}^{\prime} \cup V_{2}^{\prime}\right)\right)$ corresponding to $V_{1}^{\prime}$ and $V_{2}^{\prime}$ respectively. Let $B$ be a convex vertical annulus between vertical Legendrian ruling curves of $T_{1}$ and $T_{2}$. 
The dividing set of $B$ contains no boundary parallel dividing curves, otherwise there would be a bypass attached vertically to $T_{1}$ or $T_{2}$ by [13, Proposition 3.18], and we could use this bypass to produce a torus with infinite slope inside $M_{c_{1}^{\prime}}$. This would be a contradiction because there are no Legendrian curves with twisting number 0 isotopic to regular fibres in $M_{c_{1}^{\prime}}$ by Lemma 3.2. After cutting $M_{c_{1}^{\prime}} \backslash\left(V_{1}^{\prime} \cup V_{2}^{\prime}\right)$ along $B$ and rounding the edges, by the Edge rounding lemma [13, Lemma 3.11] we get a convex torus $T_{3}$ with slope -1 isotopic to $\partial M_{c_{1}}$. The collar $C_{1}$ is bounded by $T_{3}$ and $\partial M_{c_{1}}$.

Now we determine the isotopy type of the contact structures $\left.\xi_{n}\right|_{V_{i}}$. We recall that $D^{2} \times S^{1}$ admits exactly two universally tight contact structures with $\# \Gamma_{\partial D^{2} \times S^{1}}=2$ if its boundary slope is lesser than -1 , and exactly one its boundary slope is -1 ; see Honda [13, Proposition 5.1(2)]. It follows from the computation of the relative Euler class of the universally tight contact structures on $T^{2} \times[0,1]$ [13, Proposition 5.1] and from the correspondence between tight contact structures on $T^{2} \times[0,1]$ and on $D^{2} \times S^{1}$ [13, Proposition 4.15] that all the basic slices in the basic slices decomposition of a universally tight contact structures have the same sign. We define the sign of a universally tight contact structure on $D^{2} \times S^{1}$ as the sign of any basic slices in its decomposition.

Proposition 3.4 The contact structures $\left.\xi_{n}\right|_{V_{i}}$ are universally tight for $i=1,2,3,4$. They are positive for $i=1,2$ and negative for $i=3,4$.

Proof $\left(V_{i},\left.\xi_{n}\right|_{V_{i}}\right)$ is universally tight because $\left(M, \xi_{n}\right)$ is universally tight and the inclusions $\iota_{i}: V_{i} \rightarrow M$ induce injective maps $\left(\iota_{i}\right)_{*}: \pi_{1}\left(V_{i}\right) \rightarrow \pi_{1}(M)$. We can assume without loss of generality that $V_{i}^{\prime}$ is contained in $V_{i}$ for $i=1,2,3,4$, then $V_{i} \backslash V_{i}^{\prime}$ is the outermost basic slice in the decomposition of $V_{i}$. Since all basic slice in the decomposition of a universally tight contact structure have the same sign, the sign of $\left.\xi_{n}\right|_{V_{i} \backslash V_{i}^{\prime}}$ determines the sign of $\left.\xi_{n}\right|_{V_{i}}$. By Ghiggini-Lisca-Stipsicz [7, Lemma 2.7] the signs of $V_{1} \backslash V_{1}^{\prime}$ and of $V_{2} \backslash V_{2}^{\prime}$ are the same as the sign of $C_{1}$, and the signs of $V_{3} \backslash V_{3}^{\prime}$ and of $V_{4} \backslash V_{4}^{\prime}$ are the same as the sign of $C_{2}$. In applying [7, Lemma 2.7] we must notice that here the sign of $C_{i}$ is computed after orienting $\partial M_{c_{1}}$ by the outward normal convention, while in [7, Lemma 2.7] all boundaries are oriented by the inward normal. By a direct check on $\alpha_{2 n}\left(s_{1}, s_{2}\right)$ it easy to see that $C_{1}$ is positive and $C_{2}$ is negative.

Lemma 3.5 For $i=1, \ldots, 4$, let $V_{i}$ the neighbourhood of the singular fibre $F_{1}$ obtained by applying Lemma 3.1 to $\left(M_{c_{1}}, \widetilde{\xi}_{+}\right)$and to $\left(M_{c_{2}}, \widetilde{\xi}_{-}\right)$. Then the dividing set on a convex \#Г-minimising section of the backgrounds of $\left(M_{c_{1}}, \widetilde{\xi}_{+}\right)$and $\left(M_{c_{2}}, \widetilde{\xi}_{-}\right)$ has no boundary parallel dividing curves. 


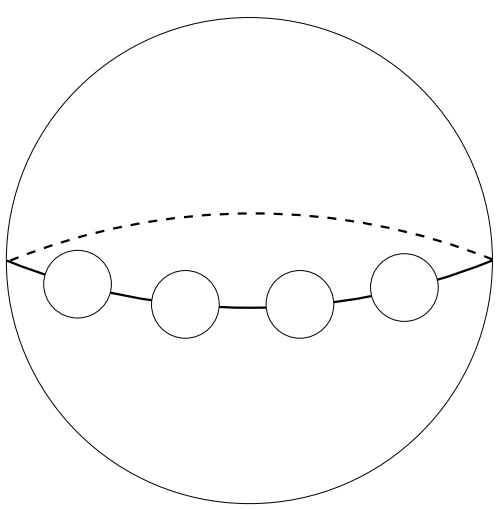

(a)

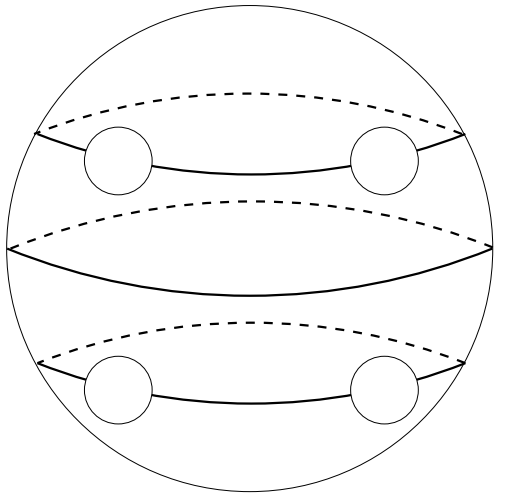

(b)

Figure 2: On the left the dividing set on a $\# \Gamma$-minimising section of the background of $\left(M, \xi_{0}\right)$. On the right the dividing set on a \#Г-minimising section of the background of $\left(M, \xi_{1}\right)$.

Proof We prove the lemma only for $\left(M_{c_{1}}, \widetilde{\xi}_{+}\right)$because the proof for $\left(M_{c_{2}}, \widetilde{\xi}_{-}\right)$is the same.

Let $C_{0}=M_{c_{1}} \backslash\left(V_{1}^{\prime} \cup V_{2}^{\prime} \cup C_{1}\right)$. Since $\left.\xi_{n}\right|_{C_{0}}=\left.\tilde{\xi}_{+}\right|_{C_{0}}$ is a tight contact structure with boundary slopes -1 and without Legendrian curves with twisting number 0 isotopic to fibres, and $\left(C_{1}, \widetilde{\xi}_{+}\right)$is a basic slice with boundary slopes -1 and $\infty$, we apply [7, Lemma 2.7] and conclude that the dividing set on a convex \#Г-minimising section of $M_{c_{1}} \backslash\left(V_{1} \cup V_{2}\right)$ has no boundary parallel dividing arcs.

Proposition 3.6 Let $S$ be a four-punctured sphere, and let $S_{0} \subset S \times S^{1}$ be a convex $\# \Gamma$-minimising section in the background $\left(S \times S^{1}, \xi_{\Gamma_{S_{0}}}\right)$ of $\left(M, \xi_{n}\right)$. Then, if we choose the neighbourhoods $V_{i}$ so that $V_{1}, V_{2}$ are contained in $M_{c_{1}}$ and $V_{3}, V_{4}$ are contained in $M_{c_{2}}$, the dividing set of $S_{0}$ is isotopic to one of the following:

(1) four dividing arcs joining the components of $\partial S_{0}$ in cyclic order, as in a necklace, if $n=0$ (see Figure 2(a)), or

(2) two dividing arcs joining $V_{1}$ to $V_{2}$, two dividing arcs joining $V_{3}$ to $V_{4}$, and $2 n-1$ homotopically non trivial parallel dividing curves in-between (see Figure 2(b)).

Proof We divide $S_{0}$ into three pieces $S_{0}^{(1)} \subset M_{c_{1}}, S_{0}^{(2)} \subset T^{2} \times\left[c_{1}, c_{2}\right]$, and $S_{0}^{(3)} \subset$ $M_{c_{2}}$, so that each piece is convex with Legendrian boundary and $\# \Gamma$-minimising in its relative homology class. Here we assume that the product structure on $T^{2} \times I$ has been deformed in small neighbourhoods of $c_{1}$ and $c_{2}$ so that $T^{2} \times\left\{c_{1}\right\}$ and $T^{2} \times\left\{c_{2}\right\}$ 


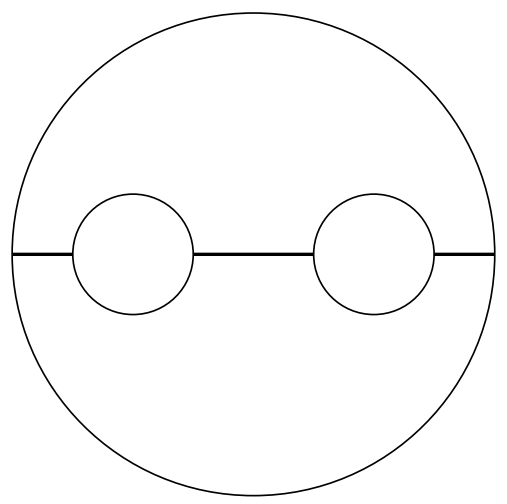

Figure 3: The dividing set on $S_{0}^{(1)}$ and $S_{0}^{(3)}$

has become convex tori with two dividing curves. If $n=0 c_{1}=c_{2}$, then we assume further that $c_{1}$ and $c_{2}$ have been replaced by $c_{1}-\epsilon$ and $c_{2}+\epsilon$, so that $T^{2} \times\left[c_{1}, c_{2}\right]$ has become the invariant neighbourhood of a convex torus.

By Lemma 3.5 the dividing set on $S_{0}^{(1)}$ and $S_{0}^{(3)}$ consists of three arcs connecting the boundary components in pairs, as in Figure 3. If $n=0 T^{2} \times\left[c_{1}, c_{2}\right]$ is an invariant neighbourhood of a convex torus, therefore the dividing set on $S_{0}^{(2)}$ consists of two arcs connecting the two components of $\partial S_{0}^{(2)}$. If $n>0$, on the other hand, by HondaKazez-Matić [15, Proposition 2.2] $S_{0}^{(2)}$ consists of a boundary parallel dividing arc for each component of $\partial S_{0}^{(2)}$ and of $2 n-1$ closed homotopically trivial curves. To see that the number of closed dividing curves is odd we glue the boundary components of $T^{2} \times\left[c_{1}, c_{2}\right]$ together by the identity map, and observe from the equation defining $\alpha_{2 n}\left(s_{1}, s_{2}\right)$ that we obtain a tight contact structure on $T^{3}$. This is possible only if the boundary parallel arcs of $\Gamma_{S_{0}^{(2)}}$ glue to give a homotopically non trivial closed curve, and this happens only if the number of closed dividing curves on $S_{0}^{(2)}$ is odd. Gluing $S_{0}^{(1)}, S_{0}^{(2)}$, and $S_{0}^{(3)}$ together we obtain the dividing set on $S_{0}$.

\section{Distinguishing the contact structures}

Our next goal is to prove that the contact manifolds $\left(M, \xi_{n}\right)$ are pairwise non isomorphic. We will follow the line of Honda-Kazez-Matić [15, Section 4.3]. For $i=1,2$ the fibre bundle $M_{i}^{\prime} \rightarrow S^{1}$ with fibre $\Sigma_{i}$ defined in Section 2 extends to a fibre bundle $M_{c_{i}} \rightarrow S^{1}$ with fibre $\bar{\Sigma}_{i}$ containing $\Sigma_{i}$. We define a surface with boundary $\widehat{\Sigma} \subset M$ as follows. Identify $T^{2} \times\left\{c_{i}\right\}$ with $T^{2}$ for $i=1,2$, and regard $\partial \bar{\Sigma}_{i}$ as a curve in $T^{2}$, 
then isotope $\bar{\Sigma}_{1}$ and $\bar{\Sigma}_{2}$ so that $\partial \bar{\Sigma}_{1}$ and $\partial \bar{\Sigma}_{2}$ minimise their geometric intersection and call $x_{1}, \ldots, x_{m}$ the intersection points between $\partial \bar{\Sigma}_{1}$ and $\partial \bar{\Sigma}_{2}$ in $T^{2}$. Then for any intersection point $x_{i}$ join $\bar{\Sigma}_{1}$ and $\bar{\Sigma}_{2}$ by a small band thickening the segment $\left\{x_{i}\right\} \times\left[c_{1}, c_{2}\right]$ so that the band intersects $T^{2} \times\{t\}$ in a linear arc whose slope is never vertical. Call the resulting surface $\widehat{\Sigma}$.

Now take two boundary-incompressible arcs $\gamma_{1} \subset \bar{\Sigma}_{1}$ and $\gamma_{2} \subset \bar{\Sigma}_{2}$ with endpoints on the same intersection points, and extend $\gamma_{1} \cup \gamma_{2}$ over the bands to get a simple closed curve $\gamma \subset \widehat{\Sigma}$. We define the framing of $\gamma$ to be the one coming from $\widehat{\Sigma}$. Let $\mathcal{L}_{n}$ be the set of the Legendrian curves in $\left(M, \xi_{n}\right)$ which are smoothly isotopic to $\gamma$, and define the maximal twisting $t\left(\mathcal{L}_{n}\right)$ to be the maximum attained by the twisting number of the curves in $\mathcal{L}_{n}$.

Proposition 4.1 $t\left(\mathcal{L}_{n}\right)=-2 n+1$.

Proof This is a corollary of [15, Proposition 4.9] once we have proved that the contact structures $\xi_{n}$ are isomorphic to the contact structures $\zeta_{2 k}$ defined in that article. To prove this we need to find a convex decomposition of $M_{c_{1}}$ and $M_{c_{2}}$ such that the dividing sets induced by $\tilde{\xi}_{+}$and $\widetilde{\xi}_{-}$on the cutting surfaces are isotopic to the dividing sets induced by the contact structures constructed by Honda, Kazez and Matić [15]. This means that we want the dividing set induced by $\widetilde{\xi}_{+}$and $\widetilde{\xi}_{-}$on any cutting surface to be boundary parallel. We will work out the details only for $\left(M_{c_{1}}, \widetilde{\xi}_{+}\right)$, because the proof for $\left(M_{c_{2}}, \widetilde{\xi}_{-}\right)$is the same.

We take $\bar{\Sigma}_{1}$ as the first cutting surface; $M_{c_{1}} \backslash \bar{\Sigma}_{1}$ is diffeomorphic to $\bar{\Sigma}_{1} \times[0,1]$, therefore we can further decompose it by cutting along discs of the form $\alpha_{i} \times I$, where $\left\{\alpha_{1}, \ldots, \alpha_{1-\chi}\left(\bar{\Sigma}_{1}\right)\right\}$ is a set of properly embedded and pairwise disjoint arcs with boundary on $\partial \bar{\Sigma}_{1}$, such that $\bar{\Sigma}_{1} \backslash\left(\alpha_{1} \cup \ldots \cup \alpha_{1-\chi}\left(\bar{\Sigma}_{1}\right)\right)$ is disc.

We can find a convex surface with Legendrian boundary isotopic to $\bar{\Sigma}_{1}$ by "patching meridional discs" of $V_{1}$ and $V_{2}$ as described in Etnyre-Honda [4, proof of Proposition 3.5] or Ghiggini-Schönenberger [9, Section 4.1]. Since the contact structures on $V_{1}$ and $V_{2}$ are both universally tight and positive, the dividing sets on the meridional discs of $V_{1}$ and $V_{2}$ consist of boundary-parallel arcs cutting out regions all with the same signs, therefore when we patch the meridional discs their dividing arcs join to give boundary-parallel dividing arcs in $\bar{\Sigma}_{1}$.

If we choose the arc $\alpha_{i}$ disjoint from the dividing set of $\bar{\Sigma}_{i}$ for all $i$, then $\partial\left(\alpha_{i} \times[0,1]\right)$ intersects the dividing set of $\partial\left(\bar{\Sigma}_{1} \times[0,1]\right)$ in exactly two points. When we make $\alpha_{i} \times[0,1]$ convex with Legendrian boundary, its dividing set consists of exactly one arc, therefore we have obtained a convex decomposition of $\left(M_{c_{1}}, \widetilde{\xi}_{+}\right)$of the required form. 
Corollary $4.2 \xi_{n}$ is not isotopic to $\xi_{m}$ if $n \neq m$

Proof $t\left(\mathcal{L}_{n}\right)$ is clearly an isotopy invariant for $\xi_{n}$.

Theorem 4.3 $\xi_{n}$ is not isomorphic to $\xi_{m}$ if $n \neq m$

Proof Let $\phi: M \rightarrow M$ be a diffeomorphism of $M$ such that $\phi_{*}\left(\xi_{n}\right)=\xi_{m}$. Denote by $T_{0}$ a fibred torus in $M$ isotopic to $\partial M_{1}^{\prime}$ and $\partial M_{2}^{\prime}$. From the equation defining $\alpha_{2 n}\left(s_{1}, s_{2}\right)$ and $\alpha_{2 m}\left(s_{1}, s_{2}\right)$ it is immediate to check that for both contact structures the isotopy class of $T_{0}$ contains a prelagrangian torus. Any other incompressible torus in $M$ non isotopic to $T_{0}$ intersect $T_{0}$ persistently, therefore by Colin [1, Theorem 1.6] any prelagrangian torus in $M$ is isotopic to $T_{0}$. This implies that $\phi\left(T_{0}\right)$ is isotopic to $T_{0}$. By Orlik [21, Theorem 8.1.7] $\phi$ is isotopic to a fibre-preserving diffeomorphism, therefore it defines an element $\bar{\phi}$ in the mapping class group of the base orbifold $B$ of $M$. Call $C_{0}$ the projection of $T_{0}$ to $B$. Since $\bar{\phi}$ fixes $C_{0}$, it must be a product of Dehn twists around $C_{0}$. Let $C_{1}$ be an essential curve in $B$ which intersects $C_{0}$ in exactly two points, and let $T_{1}$ be the pre-image of $C_{1}$ in $M . T_{1}$ is a fibred torus which splits $M$ in two submanifolds $M_{l}$ and $M_{r}$, and we may also assume without loss of generality that $F_{1}, F_{3} \subset M_{l}$ and $F_{2}, F_{4} \subset M_{r}$. Let $\phi^{\prime}$ be a composition of $\phi$ with Dehn twists around $T_{0}$ and isotopies so that $\phi^{\prime}\left(T_{0}\right)=T_{0}$ and $\phi^{\prime}\left(T_{1}\right)=T_{1}$. Then we can assume that $T_{0}$ and $T_{1}$ are fixed not only as sets, but also pointwise because the action of $\phi^{\prime}$ on the homology of $T_{0}$ and $T_{1}$ must preserve the kernels of the maps

$$
H_{1}\left(T_{0}\right) \rightarrow H_{1}\left(M_{1}^{\prime}\right), \quad H_{1}\left(T_{0}\right) \rightarrow H_{1}\left(M_{2}^{\prime}\right)
$$

and

$$
H_{1}\left(T_{1}\right) \rightarrow H_{1}\left(M_{l}\right), \quad H_{1}\left(T_{1}\right) \rightarrow H_{1}\left(M_{r}\right) .
$$

Since these kernels are linearly independent, $\phi^{\prime}$ acts trivial on $H_{1}\left(T_{0}\right)$ and $H_{1}\left(T_{1}\right)$, therefore it is isotopic to the identity.

Since $M \backslash\left(T_{0} \cup T_{1}\right)$ is a disjoint union of four solid tori and $\phi^{\prime}$ fixes their boundaries, $\phi^{\prime}$ is isotopic relative to the boundary to the identity in any of the components of $M \backslash\left(T_{0} \cup T_{1}\right)$, therefore it is isotopic to the identity on $M$. This implies that $\phi$ is isotopic to a product of Dehn twists around $T_{0}$. We may further assume that $\phi$ is supported in $M \backslash\left(M_{1}^{\prime} \cup M_{2}^{\prime}\right) \cong T^{2} \times[-1,1]$. The contact structure $\alpha_{2 n}\left(s_{1}, s_{2}\right)$ is invariant up to isotopy under Dehn twists around $T_{0}$, therefore $\xi_{m}=\phi_{*}\left(\xi_{n}\right)$ is isotopic to $\xi_{n}$. This proves that $m=n$, because $\xi_{m}$ is not isotopic to $\xi_{n}$ if $m \neq n$ by Corollary 4.2. 


\section{Ozsváth-Szabó contact invariants}

In this section we give a brief overview of those properties of Heegaard Floer homology and of the related Ozsváth-Szabó contact invariant which will be used in this article.

Heegaard Floer homology is a family of functors introduced by Ozsváth and Szabó in $[25 ; 26 ; 22]$ which, in their simplest form, associate finitely generated Abelian groups $\widehat{H F}(Y, \mathfrak{t})$ to any closed connected ${ }^{1}$ oriented $\operatorname{Spin}^{c} 3$-manifold $(Y, \mathfrak{t})$, and homomorphisms

$$
\widehat{F}_{W, \mathfrak{s}}: \widehat{H F}\left(Y_{1}, \mathfrak{t}_{1}\right) \rightarrow \widehat{H F}\left(Y_{2}, \mathfrak{t}_{2}\right)
$$

to any oriented $\operatorname{Spin}^{c}$-cobordism $(W, \mathfrak{s})$ between two $\operatorname{Spin}^{c}$-manifolds $\left(Y_{1}, \mathfrak{t}_{1}\right)$ and $\left(Y_{2}, \mathfrak{t}_{2}\right)$ such that $\left.\mathfrak{s}\right|_{Y_{i}}=\mathfrak{t}_{\mathfrak{i}}$. If we do not need to specify the Spin ${ }^{c}$-structure on $W$ we write

$$
\widehat{F}_{W}=\sum_{\substack{\left.\mathfrak{s} \in \operatorname{Spin}^{c}(W) \\ \mathfrak{s}\right|_{Y_{1}}=t_{1},\left.\mathfrak{s}\right|_{Y_{2}}=t_{2}}} \widehat{F}_{W, \mathfrak{s}} .
$$

This notation makes sense because $\widehat{F}_{W, \mathfrak{s}} \neq 0$ only for finitely many $\operatorname{Spin}^{c}$-structures $\mathfrak{s .}$

A feature of Heegaard Floer homology is that, when $Y$ is a rational homology sphere, $\chi(\widehat{H F}(Y, \mathfrak{t}))=1$ for any $\mathfrak{t} \in \operatorname{Spin}^{c}(Y)$, where the Euler characteristic is computed using a suitably defined $\mathbb{Z} / 2 \mathbb{Z}$-grading (see Ozsváth-Szabó [25, Proposition 5.1]). This implies that $\mathrm{rk} \widehat{H F}(Y, \mathfrak{t}) \geq 1$ for any $\operatorname{Spin}^{c}$-structure $\mathfrak{t}$.

Definition 5.1 A rational homology sphere $Y$ is an $L$-space if $\widehat{H F}(Y, \mathfrak{t}) \cong \mathbb{Z}$ for any $\mathfrak{t} \in \operatorname{Spin}^{c}(Y)$.

A contact structure $\xi$ on a 3 -manifold $Y$ determines a $\operatorname{Spin}^{c}$-structure $\mathfrak{t}_{\xi}$ on $Y$ such that $c_{1}\left(\mathfrak{t}_{\xi}\right)=c_{1}(\xi)$. To any contact manifold $(Y, \xi)$ we can associate an element $c(\xi) \in \widehat{H F}\left(-Y, \mathfrak{t}_{\xi}\right) / \pm 1$ which is an isotopy invariant of $\xi$, see [27]. In the following we will always abuse the notation and consider $c(\xi)$ as an element of $\widehat{H F}\left(-Y, t_{\xi}\right)$, although it is, strictly speaking, defined only up to sign. This abuse does not lead to mistakes as long as we do not use the additive structure on $\widehat{H F}\left(-Y, \mathfrak{t}_{\xi}\right)$.

Theorem 5.2 (Ozsváth-Szabó [27, Theorem 1.4 and Theorem 1.5]) If $(Y, \xi)$ is overtwisted, then $c(\xi)=0$. If $(Y, \xi)$ is Stein fillable, then $c(\xi)$ is a primitive element of $\widehat{H F}\left(-Y, \mathfrak{t}_{\xi}\right)$.

\footnotetext{
${ }^{1}$ This is the main deviation of the properties of Heegaard Floer homology from the axioms of a topological quantum field theory. We thank Tom Mrowka for pointing out this issue.
} 
Theorem 5.3 (Ozsváth-Szabó [27, Theorem 4.2], Lisca-Stipsicz [20, Theorem 2.3]) Suppose that $\left(Y^{\prime}, \xi^{\prime}\right)$ is obtained from $(Y, \xi)$ by Legendrian surgery along a Legendrian link. Then we have

$$
\widehat{F}_{W}\left(c\left(\xi^{\prime}\right)\right)=c(\xi)
$$

where $W$ is the cobordism induced by the surgery viewed as a cobordism from $-Y^{\prime}$ to $-Y$.

We observe that it makes sense to write $\widehat{F}_{W}\left(c\left(\xi^{\prime}\right)\right)$ because $\widehat{F}_{W}$ descends to a well defined map

$$
\widehat{F}_{W}: \widehat{H F}\left(-Y^{\prime}, \mathfrak{t}_{\xi^{\prime}}\right) / \pm 1 \rightarrow \widehat{H F}\left(-Y, \mathfrak{t}_{\xi}\right) / \pm 1 .
$$

There is a also a more refined version of Heegaard Floer homology called Heegaard Floer homology with twisted coefficients; see Ozsváth-Szabó [25, Section 8]. Denote by $\mathbb{Z}\left[H^{1}(Y, \mathbb{Z})\right]$ the group ring of the first cohomology group of $Y$ with integer coefficients. For any $\mathbb{Z}\left[H^{1}(Y, \mathbb{Z})\right]$-module $M$ and for any compact connected oriented 3-manifold $Y$ endowed with a $\operatorname{Spin}^{c}$-structure $\mathfrak{t}$ one can define a Heegaard Floer homology group $\widehat{H F}(Y, \mathfrak{t} ; M)$. On this Heegaard Floer homology group there is a natural structure of module over $\mathbb{Z}\left[H^{1}(Y, \mathbb{Z})\right]$ inherited by the coefficient group $M$.

The contact invariant in the context of Heegaard Floer homology with twisted coefficients is denoted by $c(\xi ; M)$. It is well defined only up to multiplication by invertibles elements of $\mathbb{Z}\left[H^{1}(Y, \mathbb{Z})\right]$, therefore it is, properly speaking, an element of the quotient $\widehat{\widehat{H F}}(Y, \mathfrak{t} ; \mathbb{Z}) / \mathbb{Z}\left[H^{1}(Y, \mathbb{Z})\right]^{*}$. If we consider $\mathbb{Z}\left[H^{1}(Y, \mathbb{Z})\right]$ as a module over itself, we write $\underline{\widehat{H F}}(Y, \mathfrak{t})$ for $\underline{\widehat{H F}}\left(Y, \mathfrak{t} ; \mathbb{Z}\left[H^{1}(Y, \mathbb{Z})\right]\right)$, and $\underline{c}(\xi)$ for $\underline{c}\left(\xi ; \mathbb{Z}\left[H^{1}(Y, \mathbb{Z})\right]\right)$. The untwisted Heegaard Floer homology group $\widehat{H F}(Y, \mathfrak{t})$ can be seen in the theory with twisted coefficients as $\underline{\widehat{H F}}(Y, \mathfrak{t} ; \mathbb{Z})$, where $\mathbb{Z}$ is considered as a trivial $\mathbb{Z}\left[H^{1}(Y, \mathbb{Z})\right]-$ module, and $c(\xi)=\underline{c}(\xi ; \mathbb{Z})$.

Remark 5.4 If $Y$ is a rational homology sphere, then $\mathbb{Z}\left[H^{1}(Y, \mathbb{Z})\right]=\mathbb{Z}$, therefore $\widehat{H F}(Y, \mathfrak{t})=\widehat{H F}(Y, \mathfrak{t})$ and $\underline{c}(\xi)=c(\xi)$.

We say that a contact manifold $(Y, \xi)$ is weakly symplectically fillable if there is a symplectic manifold $(X, \Omega)$ such that $Y=\partial X$ and $\left.\Omega\right|_{\xi}>0$. The Ozsváth-Szabó contact invariant with twisted coefficients is non trivial for weakly fillable contact manifolds. More precisely, consider the $\mathbb{Z}\left[H^{1}(Y, \mathbb{Z})\right]$-module $\mathbb{Z}[\mathbb{R}]$ generated over $\mathbb{Z}$ by the elements $T^{r}$, where $T$ is a formal variable and $r$ is any real number. A closed 2-form $\omega$ on $Y$ endows $\mathbb{Z}[\mathbb{R}]$ with the $H^{1}(Y, \mathbb{Z})$-action $\gamma \cdot T=T^{\langle\gamma \cup[\omega], Y\rangle}$. Denote by $\widehat{\widehat{H F}}(Y, \mathfrak{t} ;[\omega])$ the Heegaard Floer homology group of $(Y, \mathfrak{t})$ with twisted coefficients in $\mathbb{Z}[\mathbb{R}]$ with the module structure defined by $\omega$. 
Theorem 5.5 (Ozsváth-Szabó [24, Theorem 4.2]) Let $(Y, \xi)$ be a weakly fillable contact 3-manifold, and let $(X, \Omega)$ be one of its weak fillings. Call $\omega=\left.\Omega\right|_{Y}$, then $\underline{c}(\xi,[\omega])$ is a primitive element of $\underline{\widehat{H F}}\left(-Y, \mathfrak{t}_{\xi} ;[\omega]\right)$.

Remark 5.6 If $\omega$ is exact (in particular, if $Y$ is a rational homology sphere), then $\widehat{\widehat{H F}}\left(Y, \mathfrak{t}_{\xi} ;[\omega]\right) \cong \widehat{H F}\left(Y, \mathfrak{t}_{\xi}\right) \otimes_{\mathbb{Z}} \mathbb{Z}[\mathbb{R}]$ and $\underline{c}(\xi,[\omega])=c(\xi) \otimes 1$.

In the following we will use only the untwisted invariant because we are concerned only with rational homology spheres. We have made this excursion into Heegaard Floer homology with twisted coefficients only to show that we are not losing any generality by considering only the untwisted invariant in this article.

\section{Computation of the Ozsváth-Szabó invariants}

We construct contact structures $\zeta_{n}$ on $M\left(r_{1}, r_{2}, r_{3}\right)$ for $n \geq 0$ so that we obtain $\left(M\left(r_{1}, r_{2}, r_{3}, r_{4}\right), \xi_{n}\right)$ from $\left(M\left(r_{1}, r_{2}, r_{3}\right), \zeta_{n}\right)$ by a sequence of negative Legendrian surgeries.

The contact manifold ( $M \backslash V_{4},\left.\xi_{n}\right|_{M \backslash V_{4}}$ ) has infinite boundary slope for any $n \geq 0$. Let $\lambda$ be the unique tight contact structure with infinite boundary slope on the solid torus $D^{2} \times S^{1}$. Then $\left(M\left(r_{1}, r_{2}, r_{3}\right), \zeta_{n}\right)$ is obtained by gluing $\left(D^{2} \times S^{1}, \lambda\right)$ to $\left(M \backslash V_{4},\left.\xi_{n}\right|_{M \backslash V_{4}}\right)$ by a map $\partial D^{2} \times S^{1} \rightarrow-\partial\left(M \backslash V_{4}\right)$ represented by the identity matrix in the bases described in Section 3.

$\partial D^{2} \times S^{1} \rightarrow-\partial\left(M \backslash V_{4}\right)$

Proposition 6.1 All the contact structures $\xi_{n}$ are homotopic to $\xi_{0}$. All the contact structures $\zeta_{n}$ are homotopic to $\zeta_{0}$.

Proof All the contact structures $\xi_{n}$ coincide with $\widetilde{\xi}_{+}$on $M_{c_{1}}$, and with $\tilde{\xi}_{-}$on $M_{c_{2}}$, therefore we need to show that they are homotopic relative to the boundary on $M \backslash\left(M_{c_{1}} \cup M_{c_{2}}\right)$. We recall that $\left.\xi_{n}\right|_{M \backslash\left(M_{c_{1}} \cup M_{c_{2}}\right)}$ is a perturbation of $\left.\alpha_{2 n}\left(s_{1}, s_{2}\right)\right|_{T^{2} \times\left[c_{1}, c_{2}\right]}$ near to the boundary in order to make it convex. If we choose the functions $\varphi_{2 n}$ so that they coincide on $\left[-1, c_{1}+\epsilon\right]$ and on $\left[c_{2}-\epsilon, 1\right]$, and do the perturbations in $\left[-1, c_{1}+\frac{\epsilon}{2}\right] \cup\left[c_{2}-\frac{\epsilon}{2}, 1\right]$, we only need to show that all $\alpha_{2 n}$ are homotopic through a homotopy which is constant in $\left[-1, c_{1}+\epsilon\right] \cup\left[c_{2}-\epsilon, 1\right]$.

In order to construct such a homotopy, take a cut-off function $\beta$ which is 0 on the union $\left[-1, c_{1}+\frac{\epsilon}{2}\right] \cup\left[c_{2}-\frac{\epsilon}{2}, 1\right]$ and 1 on $\left[c_{1}+\epsilon, c_{2}-\epsilon\right]$. For any $n_{0}$ and $n_{1}$ we can 
take a number $K \gg 0$ which is big enough so that, for any $\lambda \in[0,3]$, the kernel of the 1-form $H_{\lambda}$ defined by

$$
H_{\lambda}=\left\{\begin{array}{l}
\lambda K \beta(t) d t+\left(\cos \left(\varphi_{2 n_{0}}(t)\right) d x+\sin \left(\varphi_{2 n_{0}}(t)\right) d y\right) \quad \text { if } \quad \lambda \in[0,1] \\
K \beta(t) d t+(2-\lambda)\left(\cos \left(\varphi_{2 n_{0}}(t)\right) d x+\sin \left(\varphi_{2 n_{0}}(t)\right) d y\right)+ \\
(\lambda-1)\left(\cos \left(\varphi_{2 n_{1}}(t)\right) d x+\sin \left(\varphi_{2 n_{1}}(t)\right) d y\right) \quad \text { if } \quad \lambda \in[1,2] \\
(3-\lambda) K \beta(t) d t+\left(\cos \left(\varphi_{2 n_{1}}(t)\right) d x+\sin \left(\varphi_{2 n_{1}}(t)\right) d y\right) \text { if } \lambda \in[2,3]
\end{array}\right.
$$

is a tangent 2-plane field. A smoothing of $H_{\lambda}$ provides the wanted homotopy. The homotopy between $\zeta_{n_{0}}$ and $\zeta_{n_{1}}$ follows at once because $\left(N, \zeta_{n_{0}}\right)$ and $\left(N, \zeta_{n_{1}}\right)$ are obtained by modifying $\left(M, \xi_{n_{0}}\right)$ and $\left(M, \xi_{n_{1}}\right)$ in $M_{c_{2}}$ where the homotopy $H_{\lambda}$ is constant.

Proposition 6.2 $\left(M\left(r_{1}, r_{2}, r_{3}, r_{4}\right), \xi_{n}\right)$ is obtained from $\left(M\left(r_{1}, r_{2}, r_{3}\right), \zeta_{n}\right)$ by a sequence of Legendrian surgeries.

Proof $\left(D^{2} \times S^{1}, \lambda\right)$ is diffeomorphic to a standard neighbourhood of a Legendrian curve with twisting number 0 . Moreover the core of $D^{2} \times S^{1}$ is isotopic in $M\left(r_{1}, r_{2}, r_{3}\right)$ to a regular fibre, therefore $\left(M \backslash V_{4},\left.\xi_{n}\right|_{M \backslash V_{4}}\right.$ ) is the complement of the standard neighbourhood of a regular fibre with twisting number 0 in $M\left(r_{1}, r_{2}, r_{3}\right)$. This implies that $\left(M\left(r_{1}, r_{2}, r_{3}, r_{4}\right), \xi_{n}\right)$ is obtained from $\left(M\left(r_{1}, r_{2}, r_{3}\right), \zeta_{n}\right)$ by rational contact surgery. Since we have performed the surgery on a Legendrian curve with twisting number 0 , the contact surgery coefficient is equal to the smooth surgery coefficient, which is $-\frac{1}{r_{4}}<0$. By Ding-Geiges [3, Proposition 3] any contact surgery with negative coefficient can be expanded into a sequence of Legendrian surgeries.

Proposition 6.3 $\left(M\left(r_{1}, r_{2}, r_{3}\right), \zeta_{0}\right)$ is Stein fillable. $\left(M\left(r_{1}, r_{2}, r_{3}\right), \zeta_{n}\right)$ is overtwisted for $n \geq 1$.

Proof The background of $\left(N, \zeta_{n}\right)$ is isomorphic to a $S^{1}$-invariant contact structure in $S^{\prime} \times S^{1}$ where $S^{\prime}$ is a three-punctured sphere. A convex \#Г-minimising section $S_{0}^{\prime}$ of the background of $\left(N, \zeta_{n}\right)$ is obtained by gluing a meridional disc of $\left(D^{2} \times S^{1}, \lambda\right)$ to $S_{0}$ along the component of $\partial S_{0}$ corresponding to $V_{4}$. The dividing set of $S_{0}^{\prime}$ consists of

(1) three arcs joining the boundary components of $\partial S_{0}^{\prime}$ in pairs when $n=0$ (Figure 4(a)), or 


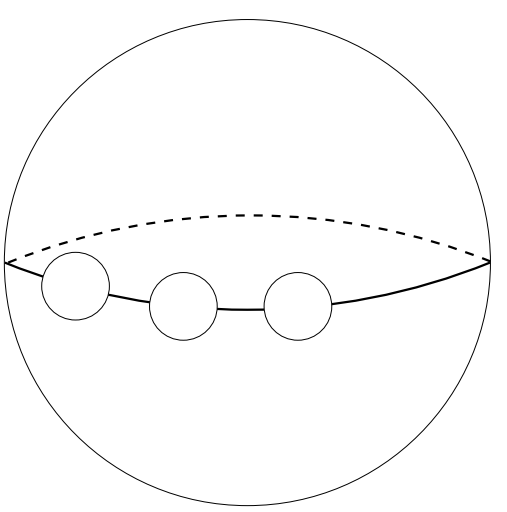

(a)

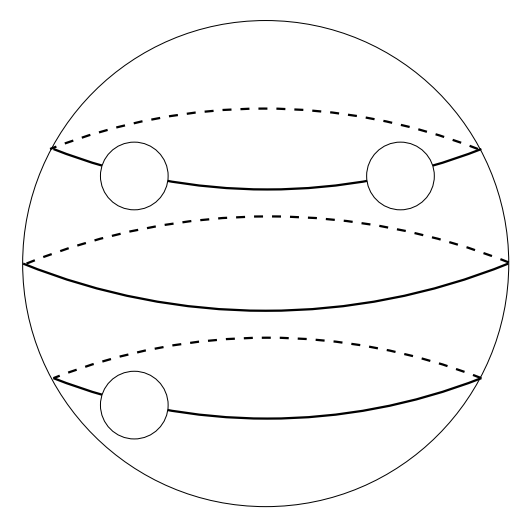

(b)

Figure 4: On the left the dividing set on a $\# \Gamma$-minimising section of the background of $\left(N, \zeta_{0}\right)$. On the right the dividing set on a $\# \Gamma$-minimising section of the background of $\left(N, \zeta_{1}\right)$.

(2) two arcs joining two boundary components, one arc with both endpoints on the third boundary component, and $2 n-1$ closed curves parallel to the third boundary component when $n \geq 1$ (Figure 4(b)).

We consider $\zeta_{0}$ first. It has been proved by Ghiggini, Lisca and Stipsicz [8] that any tight contact structure on $N=M\left(r_{1}, r_{2}, r_{3}\right)$ which is isomorphic to the background of $\left(N, \zeta_{0}\right)$ in the complement of tubular neighbourhoods of the singular fibres is Stein fillable independently of its restrictions to the neighbourhoods of the singular fibres, provided that the restrictions are tight.

Now we consider $\zeta_{n}$ for $n \geq 1$. The dividing arc with both endpoints on $-\partial\left(N \backslash V_{3}\right)$ produces a singular bypass on $S_{0}^{\prime}$ by Honda [13, Proposition 3.18]. By [13, Lemma 3.15] attaching this bypass to $-\partial\left(N \backslash V_{3}\right)$ we thicken $V_{3}$ to $V_{3}^{\prime}$ so that $-\partial\left(M \backslash V_{3}^{\prime}\right)$ has slope 0 .

Take a point $p$ belonging to another dividing curve of $S_{0}^{\prime}$, then $\{p\} \times S^{1}$ is a Legendrian fibre with twisting number 0 because $\left.\zeta_{n}\right|_{N \backslash\left(V_{1} \cup V_{2} \cup V_{3}\right)}$ is $S^{1}$-invariant by Honda [14, Section 4.3]. Applying the Imbalance principle [13, Proposition 3.17] we use this curve to find a vertical bypass attached to $\partial\left(N \backslash V_{3}^{\prime}\right)$. The attachment of this bypass gives a further thickening of $V_{3}^{\prime}$ to $V_{3}^{\prime \prime}$ so that $-\partial\left(N \backslash V_{3}^{\prime \prime}\right)$ has infinite boundary slope again. By [13, Proposition 4.16] there is a standard torus with slope $-r_{3}$ in $V_{3}^{\prime \prime} \backslash V_{3}$. This torus produces an overtwisted disc in $\left(N, \zeta_{n}\right)$.

Corollary 6.4 $\left(M\left(r_{1}, r_{2}, r_{3}, r_{4}\right), \xi_{0}\right)$ is Stein fillable. 
Theorem 6.5 $c\left(\xi_{n}\right)=0$ for $n \geq 1$.

Proof The contact structures $\xi_{n}$ are homotopic for $n \geq 0$ by Proposition 6.1, in particular they determine the same $\operatorname{Spin}^{c}$-structure on $M$. The same is true for the contact structures $\zeta_{n}$ on $N$. Let $\mathfrak{t}_{\xi}$ denote the $\operatorname{Spin}^{c}$-structure on $M$ determined by the $\xi_{n}$ 's, and let $\mathfrak{t}_{\zeta}$ denote the $\operatorname{Spin}^{c}$-structure on $N$ determined by the $\zeta_{n}$ 's.

The surgery links from Proposition 6.2 are all smoothly isotopic independently of the contact structure, therefore they determine the same smooth cobordism $W$ from $-M$ to $-N$. This implies that for any $n \geq 0$ we have $\widehat{F}_{W}\left(c\left(\xi_{n}\right)\right)=c\left(\zeta_{n}\right)$. Both $-M$ and $-N$ are $L-$ spaces by Ozsváth-Szabó [23, Lemma 2.6], hence $\widehat{H F}\left(-M, \mathfrak{t}_{\xi}\right)$ is generated by $c\left(\xi_{0}\right)$ and $\widehat{H F}\left(-N, \mathfrak{t}_{\zeta}\right)$ is generated by $c\left(\zeta_{0}\right)$ because $\xi_{0}$ and $\zeta_{0}$ are Stein fillable by Corollary 6.4 and Proposition 6.3. This implies that $\widehat{F}_{W}$ is injective. $c\left(\zeta_{n}\right)=0$ when $n \geq 1$ because $\zeta_{n}$ is overtwisted by Proposition 6.3 , therefore the injectivity of $\widehat{F}_{W}$ implies that $c\left(\xi_{n}\right)=0$.

$c\left(\zeta_{n}\right)=0$ when $n \geq 1$ because $\zeta_{n}$ is overtwisted by 6.3 , therefore

Corollary 6.6 $\left(M\left(r_{1}, r_{2}, r_{3}, r_{4}\right), \xi_{n}\right)$ is not weakly symplectically fillable for $n \geq 1$.

\section{Further examples}

More examples of tight contact manifolds with trivial contact invariants can be constructed by Legendrian surgery on $\left.\left(M\left(r_{1}, r_{2}, r_{3}\right), \xi_{n}\right)\right)$ with the help of the following tightness criterion, which was implicitly proved in Hofer's work on the Weinstein conjecture for overtwisted contact structures [12].

Proposition 7.1 Let $(Y, \xi)$ be a hypertight contact manifold. Then any contact manifold $\left(Y^{\prime}, \xi^{\prime}\right)$ obtained by Legendrian surgery on $(Y, \xi)$ is tight.

Sketch of the proof Pick a contact form $\alpha$ on $Y$ whose Reeb flow has no contractible periodic orbits. The Legendrian surgery defines an exact symplectic cobordism $(W, d \lambda)$ from $(Y, \xi)$ to $\left(Y^{\prime}, \xi^{\prime}\right)$ such that $\left.\lambda\right|_{Y}=\alpha$. Extend $W$ to a non compact exact symplectic manifold $(\widehat{W}, d \widehat{\lambda})$ by gluing $\left(Y \times(-\infty, 0], d\left(e^{t} \alpha\right)\right)$ to $Y \subset W$.

Suppose that $\left(Y^{\prime}, \xi^{\prime}\right)$ is overtwisted, then there is a 2 -sphere $S$ embedded in $Y^{\prime}$ whose characteristic foliation contains a closed periodic orbit and has an elliptic point in each connected component of the complement of the periodic orbit as unique singularities. Now we proceed as in [12]: we start filling $S$ by a Bishop family of holomorphic discs 
originating from an elliptic singularity of the characteristic foliation of $S$. Since $S$ cannot be filled completely because of the periodic orbit in the characteristic foliation, we end up with a contractible periodic orbit of the Reeb flow associated to $\alpha$ exactly like in the case of the symplectisation, contradicting our hypothesis.

Isotope the singular fibres $F_{i}$ to Legendrian curves with twisting number -1 in $\left(M\left(r_{1}, r_{2}, r_{3}, r_{4}\right), \xi_{n}\right)$. Denote by $\left(M\left(r_{1}^{\prime \prime}, r_{2}^{\prime \prime}, r_{3}^{\prime \prime}, r_{4}^{\prime \prime}\right), \xi_{n}^{\prime \prime}\right)$ a contact manifold obtained by contact surgery on the singular fibres $F_{i}$ with smooth surgery coefficient $r_{i}^{\prime}$. From well known properties of rational surgery and Seifert invariants we have $-\frac{1}{r_{i}^{\prime \prime}}=-\frac{1}{r_{1}}-\frac{1}{r_{1}^{\prime}}$, therefore $r_{i}^{\prime \prime}=\frac{r_{i} r_{i}^{\prime}}{r_{i}+r_{i}^{\prime}}$. Observe that, in general, $\xi_{n}^{\prime \prime}$ is not uniquely determined by the surgery coefficients, however we will not care about this fact because our argument is independent of the choices determining $\xi_{n}^{\prime \prime}$.

If $r_{i}^{\prime}<-1$ by Ding-Geiges [3, Proposition 3] any contact surgery on $F_{i}$ with smooth surgery coefficient $r_{i}^{\prime}$ can be realised as a Legendrian surgery on a Legendrian link in $\left(M\left(r_{1}, r_{2}, r_{3}, r_{4}\right), \xi_{n}\right)$, and $\xi_{n}^{\prime \prime}$ is determined by the rotation numbers of the components of the Legendrian surgery link. By Proposition 7.1 the resulting contact manifold $\left(M\left(r_{1}^{\prime \prime}, r_{2}^{\prime \prime}, r_{3}^{\prime \prime}, r_{4}^{\prime \prime}\right), \xi_{n}^{\prime \prime}\right)$ is tight.

The proof of Theorem 6.5 goes through for this more general family of contact manifolds, therefore we have the following theorem.

Theorem 7.2 If $r_{i}^{\prime}<-1$ and $n>0$, for any choice of the rotation numbers of the components of the Legendrian surgery link defining $\xi_{n}^{\prime \prime}$ we have $c\left(\xi_{n}^{\prime \prime}\right)=0$.

Most of the contact structures $\xi_{n}^{\prime \prime}$ are virtually overtwisted. To prove this we apply Lemma 3.1 to $\left(M\left(r_{1}^{\prime \prime}, r_{2}^{\prime \prime}, r_{3}^{\prime \prime}, r_{4}^{\prime \prime}\right), \xi_{n}^{\prime \prime}\right)$ in order to find tubular neighbourhoods $V_{i}$ of the singular fibres, and observe that the restriction of $\xi_{n}^{\prime \prime}$ to $V_{i}$ may contain basic slices with different signs, depending on the choices made in the contact surgery. In those cases $\left.\xi_{n}^{\prime \prime}\right|_{V_{i}}$, and therefore $\xi_{n}^{\prime \prime}$, is virtually overtwisted.

An alternative way to prove that most contact structures $\xi_{n}^{\prime \prime}$ are virtually overtwisted is by applying Gompf's trick [10, Proposition 5.1] to the Legendrian surgery diagrams for $\left(M\left(r_{1}^{\prime \prime}, r_{2}^{\prime \prime}, r_{3}^{\prime \prime}, r_{4}^{\prime \prime}\right), \xi_{n}^{\prime \prime}\right)$ having components with non maximal rotation number in order to find an overtwisted disc in a finite cover.

The proof of Proposition 4.1 does not extend immediately to $\xi_{n}^{\prime \prime}$ because the contact structures considered by Honda-Kazez-Matić [15] are universally tight, therefore we are not able to prove that they are all distinct. However we believe that $\xi_{n}^{\prime \prime}$ and $\xi_{m}^{\prime \prime}$ are non isomorphic if $m \neq n$, and that two contact structures on $M\left(r_{1}^{\prime \prime}, r_{2}^{\prime \prime}, r_{3}^{\prime \prime}, r_{4}^{\prime \prime}\right)$ obtained by Legendrian surgery on $\left(M\left(r_{1}, r_{2}, r_{3}, r_{4}\right), \xi_{n}\right)$ are non isotopic if they are obtained by surgery on Legendrian links with different rotation numbers. 


\section{Final considerations}

Giroux has introduced the a topological invariant for 3-dimensional contact manifolds defined as follows.

Definition 8.1 Let $\xi$ be a contact structure on a 3-manifold $Y$. The torsion of $(Y, \xi)$ is the supremum of the integers $n \geq 1$ for which there exists a contact embedding

$$
\left(T^{2} \times[0,1], \operatorname{ker}(\cos (2 n \pi z) d x-\sin (2 n \pi z) d y)\right) \hookrightarrow(Y, \xi) .
$$

We declare the torsion of $(Y, \xi)$ to be 0 if no such an embedding exists.

We denote the torsion of $(Y, \xi)$ by $\operatorname{Tor}(Y, \xi)$. One can deduce from Theorem 4.3 that $\operatorname{Tor}\left(M, \xi_{n}\right)=n$, therefore Corollary 6.6 adds further evidence to the following conjecture, which the author learnt from Eliashberg.

Conjecture 8.2 If $\operatorname{Tor}(Y, \xi)>0$ then $(Y, \xi)$ is not strongly symplectically fillable.

It seems also plausible that the following stronger statement holds.

Conjecture 8.3 If $\operatorname{Tor}(Y, \xi)>0$ then $c(\xi)=0$.

Conjecture 8.3 implies Conjecture 8.2 because a strongly symplectically fillable contact manifold has non trivial contact invariant by Remark 5.6. On the other hand there are contact manifolds with positive torsion which are weakly fillable, therefore they have non trivial twisted contact invariant.

We are able to define another family of universally tight contact structures $\eta_{n}$ on $M\left(r_{1}, r_{2}, r_{3}, r_{4}\right)$ for $n \geq 0$ coinciding with $\xi_{+}$on $M^{\prime}\left(r_{1}, r_{2}\right)$ and on $M^{\prime}\left(r_{3}, r_{4}\right)$, and with $\alpha_{2 n+1}\left(s_{1}, s_{2}\right)$ on $T^{2} \times[-1,1]$. We observe that $\operatorname{Tor}\left(M, \eta_{n}\right)=n$. Since all the example of tight contact structures with trivial Ozsváth-Szabó invariants we know at present have positive torsion, it would be interesting to compute $c\left(\eta_{0}\right)$. Unfortunately the strategy adopted in this article fails for computing $c\left(\eta_{n}\right)$, because we cannot show that the $\eta_{n}$ 's are homotopic to Stein fillable contact structures.

\section{References}

[1] V Colin, Sur la torsion des structures de contact tendues, Ann. Sci. École Norm. Sup. (4) 34 (2001) 267-286 MR1841879

[2] V Colin, K Honda, Constructions contrôlées de champs de Reeb et applications arXiv:math.GT/0411640 
[3] F Ding, H Geiges, A Legendrian surgery presentation of contact 3-manifolds, Math. Proc. Cambridge Philos. Soc. 136 (2004) 583-598 MR2055048

[4] J B Etnyre, K Honda, Tight contact structures with no symplectic fillings, Invent. Math. 148 (2002) 609-626 MR1908061

[5] J B Etnyre, L L Ng, Problems in low dimensional contact topology, from: "Topology and geometry of manifolds (Athens, GA, 2001)", Proc. Sympos. Pure Math. 71, Amer. Math. Soc., Providence, RI (2003) 337-357 MR2024641

[6] P Ghiggini, Ozsváth-Szabó invariants and fillability of contact structures, Math. Z. 253 (2006) 159-175

[7] P Ghiggini, P Lisca, A I Stipsicz, Classification of tight contact structures on some small Seifert 3-manifolds arXiv:math.SG/0509714

[8] P Ghiggini, P Lisca, A I Stipsicz, Classification of tight contact structures on small Seifert 3-manifolds with $e_{0} \geq 0$, Proc. Amer. Math. Soc. 134 (2006) 909-916 MR2180909

[9] P Ghiggini, S Schönenberger, On the classification of tight contact structures, from: "Topology and geometry of manifolds (Athens, GA, 2001)", Proc. Sympos. Pure Math. 71, Amer. Math. Soc., Providence, RI (2003) 121-151 MR2024633

[10] R E Gompf, Handlebody construction of Stein surfaces, Ann. of Math. (2) 148 (1998) 619-693 MR1668563

[11] A Hatcher, Notes on Basic 3-Manifold Topology

[12] H Hofer, Pseudoholomorphic curves in symplectizations with applications to the Weinstein conjecture in dimension three, Invent. Math. 114 (1993) 515-563 MR1244912

[13] K Honda, On the classification of tight contact structures. I, Geom. Topol. 4 (2000) 309-368 MR1786111

[14] K Honda, On the classification of tight contact structures. II, J. Differential Geom. 55 (2000) 83-143 MR1849027

[15] K Honda, W H Kazez, G Matić, Convex decomposition theory, Int. Math. Res. Not. (2002) 55-88 MR1874319

[16] P Lisca, G Matić, Transverse contact structures on Seifert 3-manifolds, Algebr. Geom. Topol. 4 (2004) 1125-1144 MR2113899

[17] P Lisca, A I Stipsicz, Ozsváth-Szabó invariants and tight contact three-manifolds II arXiv: math.SG/0404136

[18] P Lisca, A I Stipsicz, Ozsváth-Szabó invariants and tight contact three-manifolds III arXiv:math.SG/0505493

[19] P Lisca, A I Stipsicz, Ozsváth-Szabó invariants and tight contact three-manifolds I, Geom. Topol. 8 (2004) 925-945 MR2087073 
[20] P Lisca, A I Stipsicz, Seifert fibered contact three-manifolds via surgery, Algebr. Geom. Topol. 4 (2004) 199-217 MR2059189

[21] P Orlik, Seifert manifolds, Springer, Berlin (1972) MR0426001

[22] P Ozsváth, Z Szabó, Holomorphic triangles and invariants for smooth four-manifolds arXiv:math.SG/0110169

[23] P Ozsváth, Z Szabó, On the Floer homology of plumbed three-manifolds, Geom. Topol. 7 (2003) 185-224 MR1988284

[24] P Ozsváth, Z Szabó, Holomorphic disks and genus bounds, Geom. Topol. 8 (2004) 311-334 MR2023281

[25] P Ozsváth, Z Szabó, Holomorphic disks and three-manifold invariants: properties and applications, Ann. of Math. (2) 159 (2004) 1159-1245 MR2113020

[26] P Ozsváth, Z Szabó, Holomorphic disks and topological invariants for closed threemanifolds, Ann. of Math. (2) 159 (2004) 1027-1158 MR2113019

[27] P Ozsváth, Z Szabó, Heegaard Floer homology and contact structures, Duke Math. J. 129 (2005) 39-61 MR2153455

[28] P Scott, The geometries of 3-manifolds, Bull. London Math. Soc. 15 (1983) 401-487 MR705527

[29] W P Thurston, H E Winkelnkemper, On the existence of contact forms, Proc. Amer. Math. Soc. 52 (1975) 345-347 MR0375366

[30] H Wu, Legendrian vertical circles in small Seifert spaces arXiv:math.GT/0310034

CIRGET, Université du Québec à Montréal, Case Postale 8888, succursale Centre-Ville Montréal (Québec) H3C 3P8, Canada

ghiggini@math.uqam. ca

Proposed: Peter Ozsváth

Seconded: Tomasz Mrowka, Ronald Stern
Received: 4 November 2005

Accepted: 26 December 2005 